ORIGINAL ARTICLE

\title{
Cerebral perfusion in infants and neonates: preliminary results obtained using dynamic susceptibility contrast enhanced magnetic resonance imaging
}

\author{
S F Tanner, L Cornette, L A Ramenghi, L S Miall, J P Ridgway, M A Smith, M I Levene
}

Arch Dis Child Fetal Neonatal Ed 2003;88:F525-F530

See end of article for authors' affiliations .....................

Correspondence to: Dr Cornette, Peter Congdon Neonatal Unit, Clarendon Wing, C Floor, Leeds General Infirmary, Leeds LS2 9NS, UK; luc.cornette@lth.nhs.uk

Accepted

8 November 2002

\begin{abstract}
Background: Previous studies have used the dynamic susceptibility contrast enhanced (DSCE) magnetic resonance (MR) imaging technique to measure cerebral perfusion in adults.

Objective: To assess the feasibility of the technique in a heterogeneous cohort of sick human infants and identify cerebral perfusion abnormalities.

Methods: Perfusion measurements were made by characterising the changing concentration of an injected bolus of contrast agent using a series of MR images acquired during the first pass of the contrast bolus. Qualitative values of relative cerebral blood flow (rCBF) were then calculated from these data on a pixel by pixel basis to generate parametric maps of perfusion.

Results: Images of perfusion were successfully calculated from 12 out of 27 neonates and infants, all with established cerebral pathology. Normal vascular anatomical structures such as the circle of Willis were identified within all calculated images. Values of rCBF were generally larger in grey matter than in white matter. In several patients, perfusion abnormalities resulted in structural abnormalities which were detected in conventional MR imaging at follow up. The acquisition of perfusion data was most difficult when the least mature brains were examined because of motion artefacts and a smaller head size with a lower level of rCBF than adults.

Conclusions: This preliminary study shows that: $(a)$ maps of $\mathrm{rCBF}$ can be acquired from neonates and infants; (b) characterisation of the bolus passage becomes progressively easier as the brain matures; $(c)$ early abnormalities in cerebral perfusion may have negative prognostic implications; (d) the main difficulty when using the DSCE technique to study neonates relates to image artefacts resulting from bulk head motion.
\end{abstract}

\begin{abstract}
$\Lambda$ bnormalities in cerebral perfusion are believed to be of prime importance in a number of principal pathologies associated with the neonatal and infant brain. ${ }^{1}$ A safe and acceptable method of assessing cerebral blood flow in sick newborn infants is therefore of fundamental importance in studies of neonatal neurology.

Previous measurements of perfusion in the neonatal brain have used positron emission tomography, ${ }^{2}$ single photon emission computed tomography, ${ }^{3}$ and xenon clearance. ${ }^{4}$ These techniques involve ionising radiation, which limits their use even though the associated risks are unlikely to be high. Doppler ultrasound and near infrared spectroscopy have been used for more than 10 years, but these techniques have been slow to fulfil their promise as a continuous monitor of cerebral circulation. A number of different groups have studied cerebral perfusion in adults using MR methods that characterise the first pass of an injected bolus of contrast agent-for example, dynamic susceptibility contrast enhanced (DSCE) gradient echo magnetic resonance (MR) imaging. ${ }^{5-8}$ This technique for measuring perfusion is minimally invasive and provides data at a relatively high resolution allowing direct comparison of perfusion in different structures within the brain. However, analogous measurements made in neonates are likely to be more difficult because subjects are non-cooperative, their head size is small, their heart rate is faster, and cerebral blood flow is less than in adults. The main aim of our study therefore was to assess the feasibility of acquiring cerebral perfusion data in neonates and infants using a method based on MR imaging and an injected bolus of non-ionising contrast agent. We also wished to see if this technique was capable of identifying
\end{abstract}

abnormalities in perfusion within the immature human brain.

\section{METHODS \\ Subjects}

The perfusion measurement implemented formed part of a clinical examination protocol which had received prior approval from the local ethics committee. This protocol restricted the perfusion measurement to patients who satisfied both of the following inclusion criteria. Firstly, a perfusion measurement could only be considered after it had been decided to give contrast agent as part of the routine conventional ( $\mathrm{Tl}$ and $\mathrm{T} 2$ weighted) MR examination. Secondly, patients had to have a pre-existing intravenous line in place to allow injection of the contrast medium. These inclusion criteria precluded the study of healthy infants, and perfusion studies were therefore confined to patients with established pathology (table 1).

All examinations were made with a paediatrician present, both during the MR measurements and in the transfer of infants between the ward and the scanner. The patients were continuously monitored using electrocardiography and cutaneous oxygen saturation monitoring. Blankets wrapped around the infants kept them warm during scanning, and cotton wool in their ears reduced the effects of gradient

Abbreviations: DSCE, dynamic susceptibility contrast enhanced; $M R$, magnetic resonance; Gd-DTPA, gadopentetate dimeglumine; rCBF, relative cerebral blood flow 
Table 1 Clinical details and imaging findings of infants studied

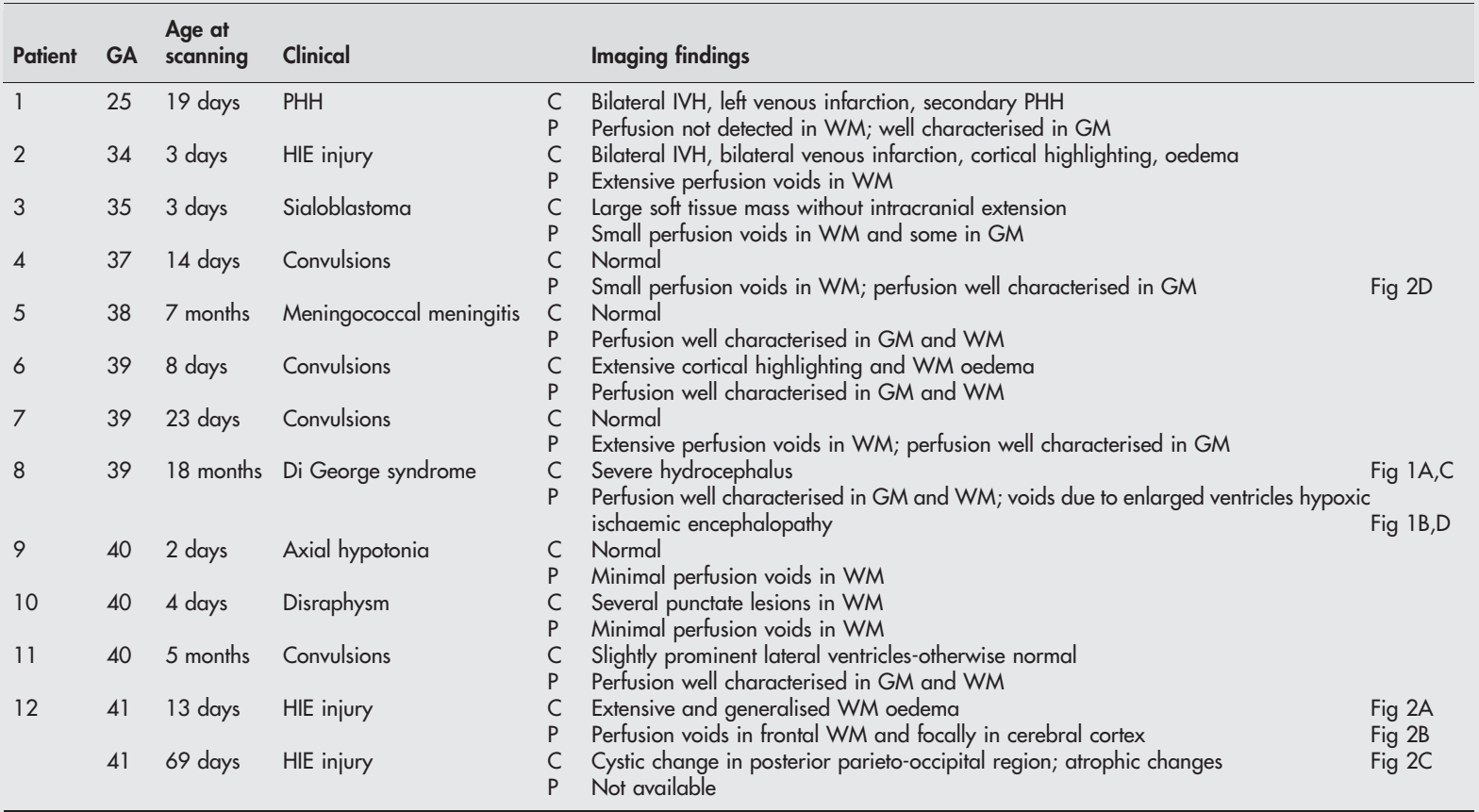

Perfusion voids correspond to pixels where the soffware was unable to calculate values of perfusion; see text.

GA, Gestational age; GM, grey matter; HIE, hypoxic ischaemic injury; ICH, intracranial haemorrhage; $\mathrm{C}$, conventional magnetic resonance imaging, $\mathrm{T} 1$ and $\mathrm{T} 2$ weighted images; $\mathrm{P}$, perfusion imaging; $\mathrm{PHH}$, post-haemorrhagic hydrocephalus; $\mathrm{WM}$, white matter.

generated noise. A pillow wrapped around the back of the head also covered the ears and protected against noise. This pillow contained polystyrene balls which restricted head movement by moulding itself to the shape of the head on removal of air. If necessary, subjects were sedated with 30 $50 \mathrm{mg} / \mathrm{kg}$ chloral hydrate administered orally. Informed parental consent was obtained before enrolment of the infants into the study.

\section{MR measurement protocol}

The MR measurement of perfusion was made using the DSCE technique which required intravenous injection of a contrast agent. Gadopentetate dimeglumine (Gd-DTPA) was manually injected at a dose of $0.1 \mathrm{mmol} / \mathrm{kg}$, followed by a $10 \mathrm{ml}$ saline injection. Injection was given over a period of five seconds or less. Perfusion measurements were made using a 1.5T Gyroscan ACS NT MR scanner (Philips Medical Systems, Best, The Netherlands) equipped with Powertrack 6000 gradients. A T2* weighted segmented gradient echo EPI sequence (TR/TE/flip/Nex $=250 \mathrm{~ms} / 25 \mathrm{~ms} / 35^{\circ} / 2$ ) was used which acquired data from seven slices in 1.5 seconds. Images were obtained with a slice thickness of $6 \mathrm{~mm}$, a field of view of $180 \mathrm{~mm}$, and a $128 \times 51$ acquisition matrix (reconstructed to $128 \times 128)$. Data were acquired in a dynamic fashion before, during, and after injection of the contrast bolus. To ensure that the magnetisation was in the steady state and that a good baseline measurement had been made, the contrast was injected after the acquisition of the 20th data set. Further 40 data sets were then obtained in the following 60 seconds to characterise the bolus passage. Conventional $\mathrm{T} 1$ and $\mathrm{T} 2$ weighted images were acquired both before and immediately after the MR perfusion measurement, and signal intensities in the brain parenchyma were compared in the two sets of images.

Parametric maps showing perfusion parameters were calculated using Philips proprietary software and techniques similar to those described by other workers. ${ }^{5-8}$ The first pass of the contrast agent resulted in a transient decrease in signal intensity within the $\mathrm{T} 2 *$ weighted MR images. This time varying signal intensity was initially converted into a series of points showing the temporal variation of contrast agent concentration for each image pixel. To allow for contrast recirculation, a gamma variate curve was then fitted to these data. This curve was used to describe the changing concentration of contrast within each pixel during the first pass of the bolus. Qualitative parameters characterising perfusion were then calculated on a pixel by pixel basis from the fitted curves to generate parametric maps of perfusion. ${ }^{7}$ The relative cerebral blood volume within each pixel was determined from the area under the gamma variate curve. The first moment of the concentration-time curve provided a measure of the mean transit time, and the relative cerebral blood flow ( $\mathrm{rCBF}$ ) was obtained from the ratio of relative cerebral blood volume to mean transit time. ${ }^{5}$

Areas where the software was able to calculate relative values of perfusion ( $\mathrm{rCBF}$ ) were then colour coded using a non-linear scale, showing differences in flow between different regions within the brain parenchyma. Perfusion voids-that is, areas where the software is not able to calculate rCBF values-correspond to pixels in which a fall in signal intensity resulting from the first pass of the contrast agent was not detected. Under these circumstances, a gamma variate function could not be fitted to the data making it impossible to calculate a value of rCBF. The black colour, indicating no discernible perfusion, was then assigned to the relevant pixels.

\section{RESULTS}

\section{Subjects}

Between August 1998 and July 2002, we obtained MR measurements of rCBF in 27 infants $(21$ born at term and six born prematurely). Of the term infants, 18 were examined in the neonatal period and three were infants with postnatal ages of 7,22 , and 30 weeks respectively. Of the six prematurely born infants, five were examined in the neonatal period, and one was a nine week old infant at the time of 
examination. Three of the prematurely born neonates were examined when their postmenstrual age was less than 37 weeks (postmenstrual age $=$ gestational age plus postnatal age).

\section{MR findings}

High quality perfusion maps were obtained from 12 out of 27 infants. Table 1 summarises the clinical reasons for scanning this group and gives details of both conventional (T1 plus T2 weighted) and functional MR techniques. In these infants, the first pass of contrast agent through the brain tissue occurred in 10-13 seconds, which appears to be slightly faster than in analogous studies of the adult brain.

The characterisation of bolus passage was not possible in 15 other infants. Image artefacts were observed in data obtained from nine of these 15 infants because of head movement, which occurred either during or just after injection of contrast. Problems with the delivery of the contrast agent prevented acquisition of perfusion data in two of 15 infants. The examination in three more infants was aborted because of technical problems with the MR scanner. Finally, in one infant, no detectable decrease in first pass signal intensity was observed in the $\mathrm{T} 2 *$ weighted images; yet, the post-contrast Tl data showed that Gd-DTPA had been successfully injected, as relative hyperintensity was seen in the choroid plexus.

In all 12 patients, relatively high levels of perfusion were observed in areas corresponding to normal vascular anatomical structures. This is shown for an 18 month old infant (fig 1). T2 weighted (fig $1 \mathrm{~A}, \mathrm{C}$ ) and perfusion (fig $1 \mathrm{~B}, \mathrm{D}$ ) images shown are obtained from two slices within the brain allowing comparison of structural and functional data. Perfusion was high in regions corresponding to the circle of Willis and the sagittal sinus.

The calculated images of perfusion $(\mathrm{rCBF})$ also showed areas of perfusion voids (table 1). As an example, fig 1D illustrates large perfusion voids corresponding to enlarged lateral ventricles. All rCBF images from the prematurely born neonates showed relatively large perfusion voids, predominantly located within the white matter, whereas significant blood flow was detected in the grey matter. Note that some of the images of the term born neonates also contained a few pixels corresponding to the white matter where rCBF values could not be calculated, whereas in the data acquired from infants who were several months old, almost all pixels corresponding to grey and white matter showed rCBF.

Figures $2 \mathrm{~A}-\mathrm{C}$ were obtained from a term born asphyxiated patient (Apgar scores 3/5/7). This is the only patient in which the parents consented to a follow up conventional MR scan. $\mathrm{T} 2$ weighted images were acquired at the age of 13 days (fig 2A) and 10 weeks (fig 2C). Figure 2A shows evidence of widespread oedema. Figure $2 \mathrm{~B}$ is an image of $\mathrm{rCBF}$ measured during the first visit; arrows indicate areas of perfusion deficit which correspond to areas of cystic degeneration seen in conventional MR at follow up (fig 2C). The perfusion image from a different 14 day old term born patient who suffered convulsions in the days just before the MR examination is included for comparison (fig 2D) and shows that the technique is, however, capable of showing perfusion in the occipital region at that age.

Our study was not intended to include clinical follow up, so there are no organised data available on outcome. Clinical data were, however, obtained by studying the medical follow up notes and by contacting the infant's general practitioner. Both asphyxiated patients (table 1), showing extensive perfusion voids, do suffer neurological morbidity (convulsions in patient 2, impaired vision, and behavioural problems in patient 12). Patient 8 (fig l, Di George and hydrocephalus) suffers severe global developmental delay. Three of the four patients with convulsions had minimal perfusion voids on functional data sets, although one of them showed extensive oedema and cortical highlighting on conventional MR. All three are currently seizure-free. However, the one patient with extensive white matter perfusion voids but normal conventional MR is still receiving anticonvulsant drugs. Patient 1 (post-haemorrhagic hydrocephalus) was extremely premature when scanned and has recently undergone ventriculoperitoneal shunt insertion. Patients 3 (sialoblastoma), 5 (meningitis), 9 (axial hypotonia), and 10 (dysraphism) had minimal perfusion voids on perfusion imaging and are neurologically normal to date.

\section{DISCUSSION}

Measurement of global cerebral blood flow in the neonate has been reported using xenon, Doppler, and near infrared spectroscopy. Regional cerebral blood flow measurements have also been attempted using positron emission tomography and single photon emission computed tomography, although in very few infants, and requiring ionising radiation. This preliminary study shows that it is possible to acquire cerebral perfusion data in human neonates and infants using a technique based on magnetic resonance (MR) imaging, with the injection of a non-ionising tracer. It also shows that the technique is sensitive to abnormality and has potential to be used clinically. We are not aware of any reports describing similar measurements in human neonates or young infants.

\section{Methodological issues}

A perfusion measurement based on the DSCE technique is only valid if several criteria are fulfilled. Firstly, one needs to show that the contrast agent remains within the intravascular compartment during the first pass of the injected bolus. ${ }^{5}$ Comparison of the Tl weighted images acquired both before and after injection of contrast indeed showed that, except for the choroid plexus, no signal enhancement was detected within the brain parenchyma for all of the patients studied after the administration of contrast agent. This observation indicates that the bulk of the injected bolus of Gd-DTPA remained within the capillary bed during the first pass of this contrast agent. It is also consistent with observations that the neonatal blood-brain barrier is intact and acts as a barrier to gadolinium chelates. ${ }^{9}$ We are therefore justified in using Gd-DTPA in a DSCE measurement of brain perfusion to examine the patients entered into this study. Secondly, no signal should be detected from non-perfused areas. The absence of blood flow in the cerebrospinal fluid and the concomitant lack of a contrast induced fall in signal intensity resulted in the analysis software correctly assigning the background colour (black) to these areas (perfusion voids). Thirdly, vascular structures such as the circle of Willis or sagittal sinus should be clearly visible as intense (red) coloured structures, representing high $\mathrm{rCBF}$, as was the case in this study.

Our study also revealed some difficulties when using the DSCE technique to measure perfusion in human infants and neonates. Firstly, ethical considerations prevented the examination of healthy subjects because the examination protocol involved an injection of a contrast agent. Measurements were therefore restricted to a heterogeneous group of patients who had well established pathology and a pre-existing intravenous line in place. This constraint prevented a comparative study involving both healthy subjects and patients, which may have facilitated the identification of any perfusion abnormalities occurring within the patient group. Secondly, and because of the above mentioned constraint, the current cohort consisted of patients with different gestational ages 


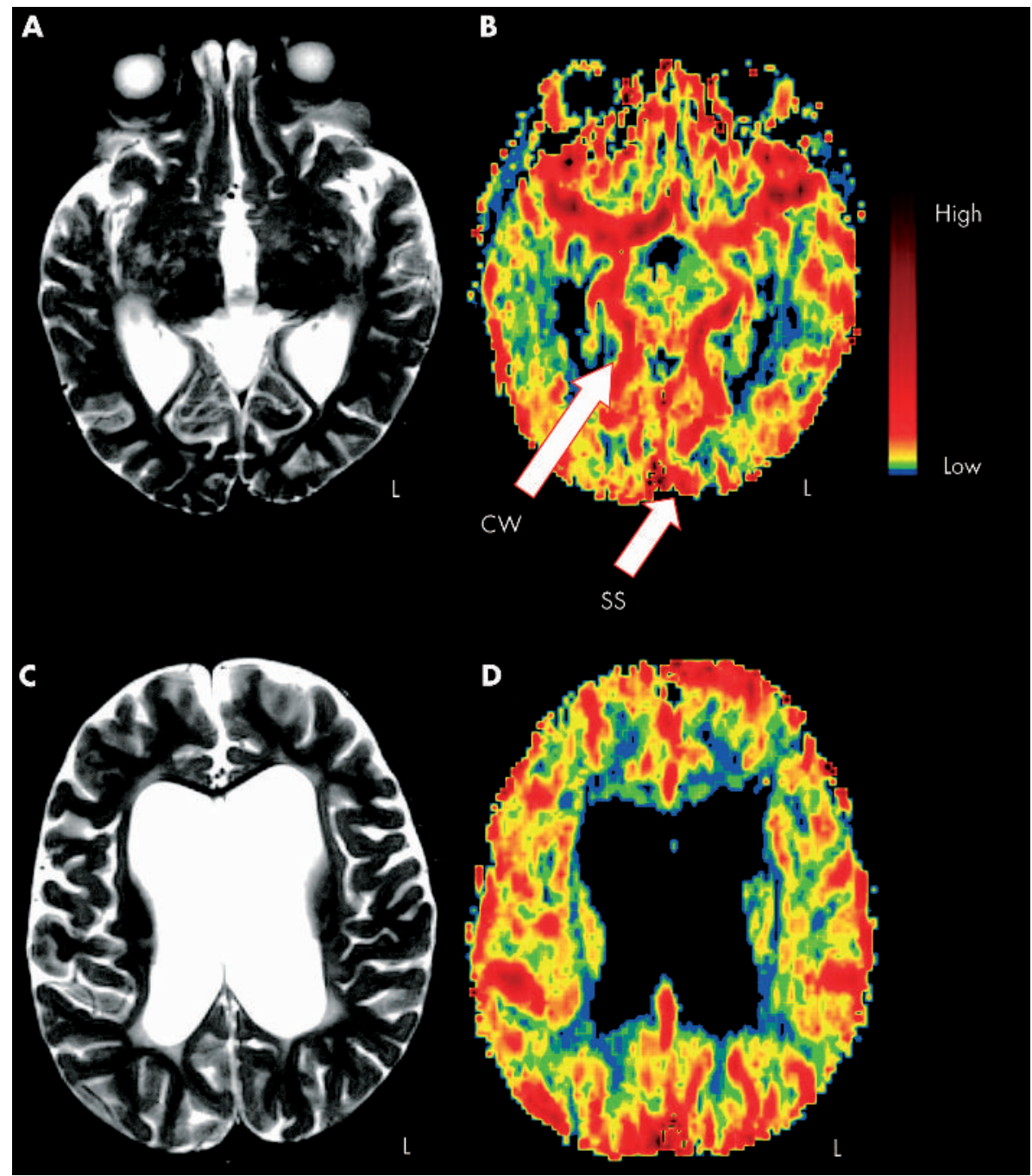

Figure 1 Magnetic resonance (MR) structural-functional comparison. Conventional T2 weighted images ( $A$ and $C$ ) versus $M R$ perfusion images ( $B$ and D); axial sections obtained at two levels of the brain. Functional data are displayed using a non-linear colour scale. CW, Circle of Willis; SS, sagittal sinus; L, left. See text for further details.

and scanned at different postmenstrual ages, impairing interpretation of time related changes in perfusion. Hence, we cannot report on brain maturation effects-that is, the evolution of rCBF-as we only obtained perfusion measurements once in each infant studied. Thirdly, in nine out of 27 infants examined, gross image artefacts were observed which corrupted the time varying signal intensity (resulting from the bolus passage) within the dynamic MR data set. Inspection of the images used in the calculations of rCBF showed that the artefacts occurred because these patients moved their heads during the first pass of the injected contrast bolus. Finally, the acquisition technique acquired only qualitative measures of perfusion or relative $\mathrm{CBF}$. We chose not to attempt quantification as this would require characterisation of the input of the contrast agent. We designed our measurement protocol to characterise the rapid first pass of the contrast agent and assumed an idealised instantaneous input of contrast agent to the brain.

\section{Interpretation of data acquired}

In spite of the difficulties outlined above, our preliminary results suggest that characterisation of the bolus passage becomes progressively easier as the brain matures. Images of rCBF acquired from older infants (fig 1B,D) are comparable to previously published data obtained from adults. In contrast, the $\mathrm{rCBF}$ data acquired from premature neonates often showed regions where it was not possible to calculate relative values of perfusion. Such perfusion voids were predominantly observed within the white matter, whereas flow in the grey matter was well detected. These observations probably reflect the lower levels of cerebral perfusion found in neonates and young infants compared with the adult brain. This finding may also confirm observations obtained from other imaging modalities, showing that blood flow to the white matter appears to be particularly low in the premature human infant (only $17 \%$ of the flow to grey matter). ${ }^{10}$ Caution is necessary, however, when interpreting the observations obtained for the current cohort of patients, as any effects on blood flow arising from their cerebral pathology or possible hypotensive episodes are unknown. It may be possible to improve the sensitivity of the rCBF measurement in prematurely born neonates by improvements in scanner hardware, the use of different TEs, or larger doses of contrast agent. This is being investigated.

The perfusion maps acquired in newborn infants are similar to those obtained from the healthy adult brain in that higher blood flow rates were observed in the cerebral cortex than in the white matter. 


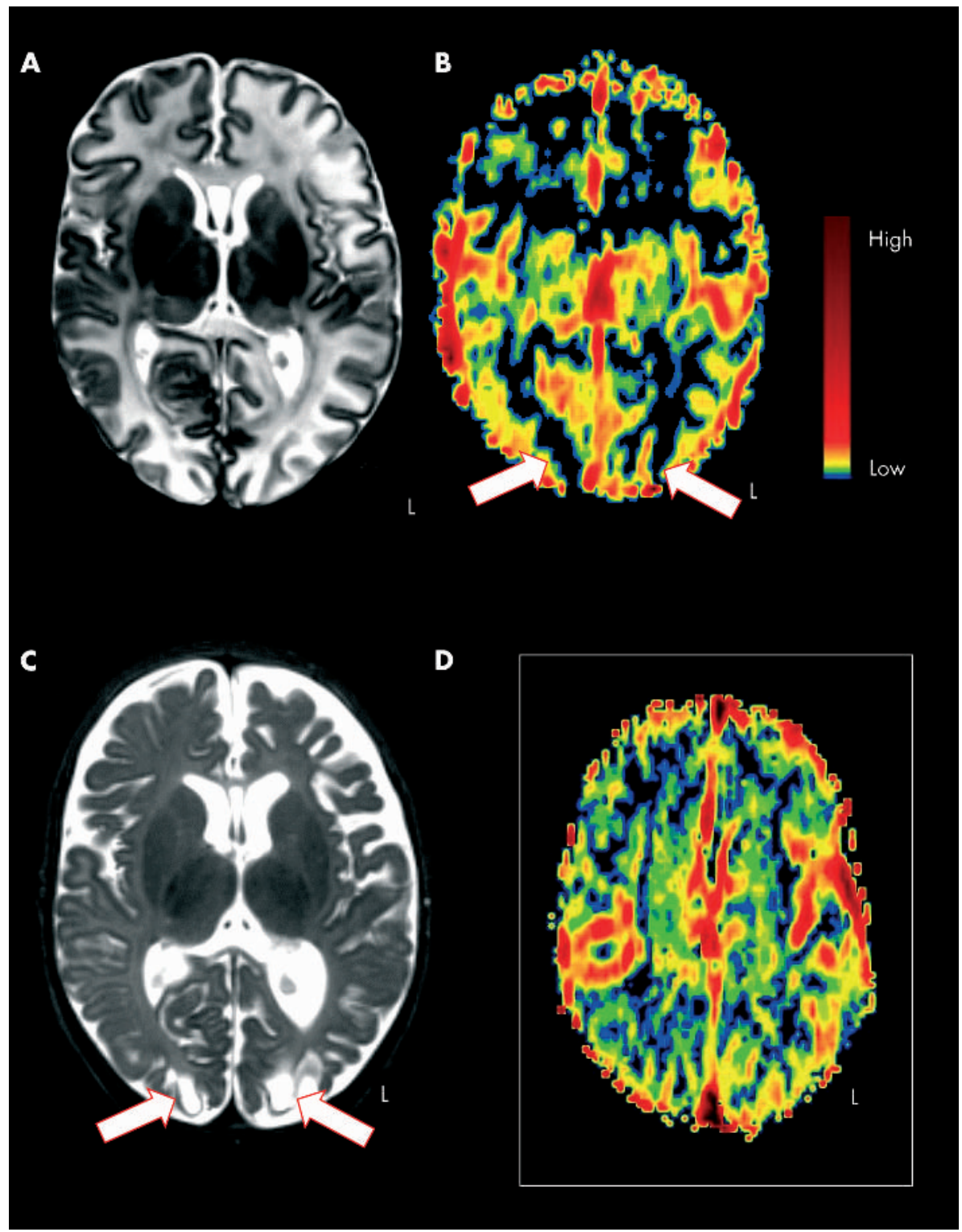

Figure 2 Magnetic resonance (MR) structural-functional comparison in hypoxic ischaemic encephalopathy. Conventional T2 weighted images (A and C) versus MR perfusion images (B and D); axial sections obtained at two levels of the brain. Colour conventions as in fig 1. Arrows indicate regions of hypoperfusion in the occipital cortex at the age of 13 days (B) corresponding to cystic lesions in the conventional MR at the age of 10 weeks (C). A-C were acquired from a patient with hypoxic ischaemic encephalopathy, whereas D was obtained from a different patient with convulsions. See text for further details.

Finally, we have shown that perfusion voids detected early in life in term newborn infants may be associated with a poor neurodevelopmental outcome. Whereas oedema was the only abnormality detected in the initial $\mathrm{T} 2$ weighted data of an asphyxiated infant (fig 2A), the image of rCBF obtained during the same neonatal period showed evidence of a perfusion deficit both bilaterally in the prefrontal cortex and posterior to the occipital horns of the lateral ventricles (fig 2B). These areas of hypoperfusion may reflect the severe hypoxic-ischaemic brain damage resulting from the insult. The posterior perfusion deficits correspond to the occipital cystic lesions seen on follow up, whereas the frontal perfusion deficit possibly precedes the frontal brain atrophy (fig 2C). We included, for comparative reasons, the $\mathrm{rCBF}$ image from a different 14 day old neonate who was suffering from fits in the days just before the MR examination and in whom no obvious regions of perfusion deficits were characterised (fig 2D). We are therefore confident that the areas indicated by arrows (fig 2B) correspond to regions of hypoperfusion, as perfusion was relatively easy to detect elsewhere within the cerebral cortex. Where extensive perfusion voids were detected, however, this may have reflected the characteristics of the delivery of the contrast bolus, and caution is required when interpreting the data. Further comparative measurements involving both healthy subjects and patients may have increased the number of perfusion abnormalities detected in this study.

In summary, the rCBF images provide data at a sufficiently high resolution to allow differences in perfusion to be detected in different regions within the brain. Our preliminary study suggests that artefacts arising from head motion still present considerable obstacles in DSCE perfusion measurements, particularly during examination of the preterm neonatal brain. In spite of the difficulties mentioned, the results show that it is possible to identify perfusion 
abnormalities. We believe that this type of measurement holds great promise for future studies of normal and abnormal brain function in a variety of illnesses. Early identification of newborns that may later develop a neurodevelopmental handicap is important in order to counsel parents and to guide further treatment. It is our hope that the technique of perfusion MR will both elucidate the complexities of the developing brain and help to develop more effective treatments for brain injury in the newborn infant.

\section{ACKNOWLEDGEMENTS}

We are much indebted to all staff of the neonatal unit and the MR department for their assistance. This work was supported by a grant from the special Trustees of the Leeds General Infirmary.

\section{Authors' affiliations}

S F Tanner, J P Ridgway, M A Smith, Academic Unit of Medical Physics and Centre of Medical Imaging Research, University of Leeds, The Wellcome Wing, Leeds General Infirmary, Great George St, Leeds LS1 3EX, UK

L Cornette, L A Ramenghi, L S Miall, M I Levene, Academic Unit of Paediatrics, Obstetrics and Gynaecology, D Floor, Clarendon Wing, Leeds General Infirmary, Leeds LS2 9NS, UK

\section{REFERENCES}

1 Floodmark $\mathrm{O}$. Imaging of the neonatal brain. In: Levene MI, Lilford RJ, eds. Fetal and neonatal neurology and neurosurgery. Edinburgh: Churchill Livingstone, 1995:105-28.

2 Volpe JJ, Herscovitch P, Perlman JM, et al. Positron emmision tomography in the newborn: extensive impairment of regional cerebral blood flow with intraventricular haemorrhage and hemorrhagic intracerebral involvement. intraventricular haemorrhage
Pediatrics 1983;72:589-601.

3 Tokumaru AM, Barkovich AJ, O'uchi T, et al. The evolution of cerebral blood flow in the developing brain: evaluation with lodine-123 lodoamphetamine SPECT and correlation with MR Imaging. AJNR Am J Neuroradiol 1999:20:845-52.

4 Greisen G, Johansen K, Ellison PH, et al. Cerebral blood flow in the newborn infant: comparison of Doppler ultrasound and ${ }^{133}$ xenon clearance. J Pediatr 1984;3:41 1-18.

5 Rempp KA, Brix G, Wenz F, et al. Quantification of regional cerebral blood flow and volume with dynamic susceptibility contrast-enhanced MR imaging. Radiology 1994;193:637-41.

6 Gore JC, Majumdar S. Measurement of tissue blood flow using intravascular relaxation agents and magnetic resonance imaging. Magn Reson Med $1990 ; 14: 242-8$

7 Reith W, Heiland S, Erb G, et al. Dynamic contrast-enhanced T2*-weighted MRI in patients with cerebrovascular disease. Neuroradiology 1997;39:250-7.

8 Calamante F, Thomas DL, Pell GS, et al. Measuring cerebral blood flow using magnetic resonance imaging techniques. J Cereb Blood Flow Metab 1999;19:701-35

9 Barkovich AJ, Latal-Hajnal B, Partridge JC, et al. MR contrast enhancement of the normal neonatal brain. AJNR Am J Neuroradiol 1997;18:1713-17.

10 Greisen G, Borch K. White matter injury in the preterm neonate: the role of perfusion. Dev Neurosci 2001;23:209-12.

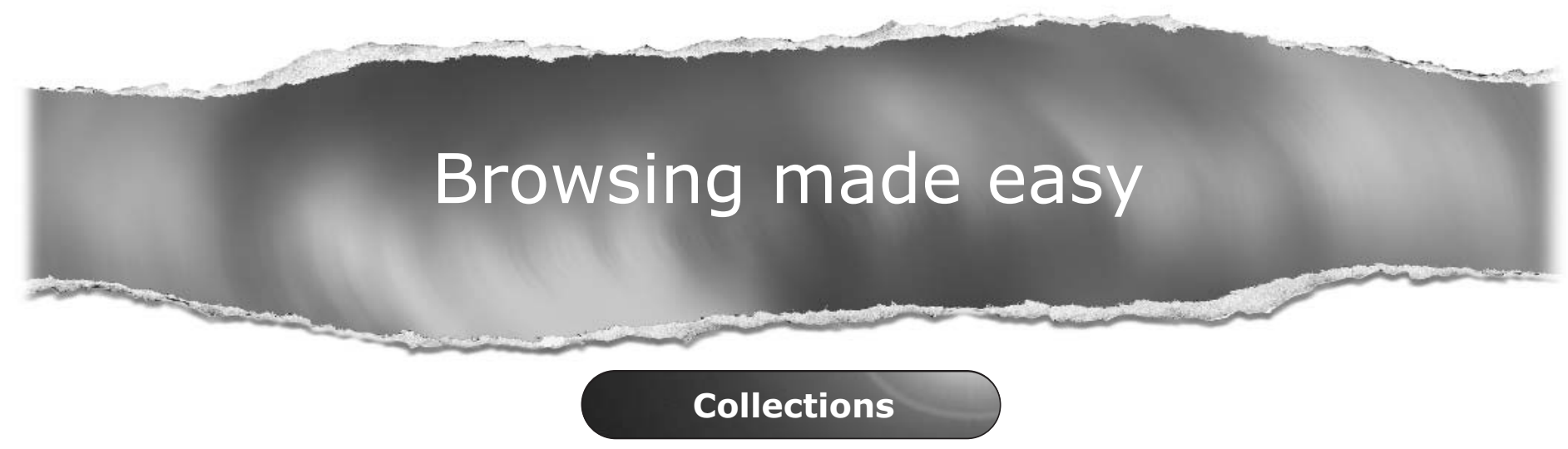

With a single click Collections allows you to find all articles that have been published in your chosen subject. Select from over 200 clinical and non-clinical topic collections and/or cross search other specialist journals, the BMJ and Cochrane Reviews

\section{www.archdischild.com}

